\title{
CD73, CD90, CD105 and Cadherin-11 RT-PCR Screening for Mesenchymal Stem Cells from Cryopreserved Human Cord Tissue
}

\author{
Hung Pham ${ }^{1,2,3}$, Richard Tonai ${ }^{1}$, Miya $\mathrm{Wu}^{4}$, Chiara Birtolo ${ }^{5}$, Monica Chen ${ }^{1}$ \\ ${ }^{I}$ Processing Laboratory, StemCyte International Cord Blood Therapeutic Company, Baldwin Park, CA \\ ${ }^{2}$ Department of Medicine, University of California - Los Angeles, Los Angeles, CA \\ ${ }^{3}$ Department of Medicine, Veterans Affair, Greater Los Angeles Healthcare System, Los Angeles, CA \\ ${ }^{4}$ Tissue Laboratory, StemCyte, New Taipei City Linkou District, Taiwan \\ ${ }^{5}$ University of Bologna, Sant' Orsola-Malpighi Hospital, Bologna, Italy
}

Background and Objectives: Mesenchymal stem cells (MSCs) are self-renewing, non-specialized cells used clinically in tissue regeneration and sourced from bone marrow, peripheral blood, umbilical cord blood and umbilical cord tissue (UCT). To demonstrate an alternative method for MSC detection, cryopreserved UCT and expanded MSC were screened for MSC markers CD73, CD90, CD105 and CDH-11 by RT-PCR.

Methods and Results: Human UCT were washed, sectioned, cryopreserved with 10\% DMSO and stored in the vapor phase of liquid nitrogen. Fresh and frozen UCT samples were expanded for MSC. UCT and MSC were processed for RNA and screened for CD73, CD90, CD105 and CDH-11 mRNA by RT-PCR. CD73, CD90 and CD105 were detected by flow cytometry and CDH-11 was detected by Western blotting. Short and long-term frozen UCT shows a loss of mRNA expression for CD73 at $33.2 \pm 34.0 \%, \mathrm{CD} 90$ at $6.2 \pm 8.2 \%, \mathrm{CD} 105$ at $17.7 \pm 21.6 \%$ and $\mathrm{CDH}-11$ at $30.1 \pm 26.7 \%$ but was not statistically significant to indicate the deterioration. Expanded MSCs from fresh UCT expressed $0.09 \pm 0.07$-fold CD73, $0.17 \pm 0.11$-fold CD90, 0.04 \pm 0.06 -fold CD105 and 0.14 \pm 0.08 -fold CDH-11. Expanded MSCs from frozen UCTs expressed $0.09 \pm 0.06$-fold CD73, $0.13 \pm 0.06$-fold CD90, $0.04 \pm 0.05$-fold CD105 and $0.11 \pm 0.06$-fold CDH-11 and confirmed by flow cytometry and Western blotting.

Conclusion: CD73, CD90, CD105 and CDH-11 were detected by RT-PCR in cryopreserved UCT and MSC expansion. $\mathrm{CDH}-11$ appears as a useful single target MSC marker for quick screening.

Keywords: CD73, CD90, CD105, Cadherin-11, Mesenchymal stem cells, Umbilical cord tissue

Received: March 2, 2017, Revised: March 21, 2018,

Accepted: April 8, 2018, Published online: May 30, 2018

Correspondence to Monica Chen

Processing Laboratory, StemCyte International Cord Blood Therapeutic Company, 13800 Live Oak Avenue, Baldwin Park, $91706 \mathrm{CA}$

Tel: +1-626-646-2499, Fax: +1-626-430-5795

E-mail: mchen@stemcyte.com

(a) This is an open-access article distributed under the terms of the Creative Commons Attribution Non-Commercial License (http://creativecommons.org/ licenses/by-nc/4.0/), which permits unrestricted non-commercial use, distribution, and reproduction in any medium, provided the original work is properly cited.

Copyright (c) 2018 by the Korean Society for Stem Cell Research

\section{Introduction}

Stem cells encompass a broad category of cells defined by their characteristics of self-renewal, non-specialization and differentiation capability (1). These characteristics constitute the cornerstone for the ongoing development of stem cell use in regenerative therapies, with several clinical phase II/III trials in progress (2). Indeed, one particular subset of stem cells, the hematopoietic stem cells (HSC), has been successfully demonstrated to regenerate the hematopoietic system using first bone marrow transplantation and in recent years, using cord blood transplantation (3). Building on the success of the hema- 
topoietic system, the optimism for repair and regeneration of other tissue types have propelled the search for other subsets of stem cells (4). Interestingly, the original source of HSCs also hosts several different subsets of stem cells capable of differentiating to other tissue types (5). These non-hematopoietic, self-renewing, non-specialized cells were isolated from bone marrow and described as possessing the ability to differentiate to bone, fat, muscle and connective tissue (5). These cells, classified as mesenchymal stem or stromal cells (MSCs), possess the differentiation capacity for, but not limited to, the adipogeinc, chrondrogenic and osteogenic lineages (6).

Clinical applications involving MSCs include tissue regeneration of smooth muscle in myocardial ischemia and myocardial infarction (7). MSCs are also involved in immunomodulation, especially in graft-versus-host disease (GVHD) (8). MSCs can be sourced from several types of tissue, including bone marrow, peripheral blood, umbilical cord blood and umbilical cord. While MSCs have been isolated from umbilical cord blood, the percentage is extremely low and umbilical cord tissue (UCT) offers a greater source (9). As a therapeutic biological product, minimal regulations have been set for MSC derived from UCT. However, several cord blood banks have developed and described methodologies for the isolation and identification of MSC from UCT (10). These methodologies vary widely, and include both mechanical and automated separation of MSC from UCT (10). Some methods utilize the enzymatic digestion of UCT for isolating MSCs, and thus, introduce xenobiotic reagents that may contaminate the final biological product (11).

Because MSC can be isolated from multiple tissue types, standardizing a universal method may not be ideal for maximizing recovery of MSCs from the source. However, standardizing the characterization of MSCs is essential due to diverse tissue procurement and methodologies of isolation (11). Current industry standards set minimal MSC requirements for identification and characterization of surface adhesion markers CD73, CD90, CD105, CD44 and CD29 coupled with the absence of hematopoietic markers CD34 and CD45 when sourcing from cord tissue (12). Immunological profiling for MSC by flow cytometry has been indispensable using MSC markers CD90, CD73 and CD105 for comparison between different cord tissue and in vitro MSC expansion (13).

However, isolation and expansion of MSC is cost prohibitive with current methodologies and identifying in vitro MSCs requires additional time for propagating the necessary number of cells for flow cytometry characterization studies (14). Exploration of methodologies to ex- pedite the detection of MSCs in cryopreserved cord tissue samples includes real time-polymerase chain reaction (RT-PCR) and alternative MSC markers. The involvement of MSC migration and adhesion from the tissue onto the plastic culture plates suggests that cellular migration and adhesion molecules as possible targets distinct from hematopoietic stem cells. Interestingly, the cell adhesion type II surface molecule, cadherin-11 (CDH-11), was discovered highly expressed in migrating mesenchymal cells whereas CDH-11 siRNA knock-down suppressed cellular migration and invasion (15). CDH-11 has been detected in circulating metastatic cells from patient blood samples by RT-PCR (16). These reports suggest CDH-11 could be employed as a single target detection for MSC in cryopreserved cord tissue by RT-PCR.

Since MSCs possess evolving therapeutic applications, we demonstrate the presence of MSCs in cryopreserved umbilical cord tissue stored in the vapor phase of liquid nitrogen. Specifically, cryopreserved UCT tests positive for MSC markers CD73, CD90, CD105 and CDH-11 by RT-PCR. Furthermore, frozen sections of thawed UCT processed for in vitro MSC expansion expressed CD73, CD90, and CD105 using flow cytometry and RT-PCR. These in vitro expanded MSCs also express CDH-11 by RT-PCR and Western blotting. Quick analysis for the presence of MSCs in cryopreserved UCT sections in long-term liquid nitrogen vapor phase storage can be achieved using RT-PCR for current MSC markers or alternatively, a CDH-11 RT-PCR screen may satisfy this requirement.

\section{Materials and Methods}

\section{Materials}

CD73, CD90 and CD105 antibodies for flow cytometry were acquired from Becton-Dickinson Biosciences (BD Stemflow, Piscataway, NJ). CDH-11 antibody (Life Technologies, Carlsbad, CA), horseradish peroxidase-conjugated anti-rabbit $\mathrm{IgG}$ or anti-mouse $\mathrm{IgG}$ and ECL reagents were acquired from Thermo Fisher Scientific (Waltham, MA). GAPDH antibody was acquired from Santa Cruz Biotechnology (Santa Cruz, CA). CryoStor CS10 was obtained from Stemcell Technologies (Vancouver, BC). Cellgro phosphate-buffered saline solution (PBS) without calcium or magnesium was obtained from Corning (Corning, NY). DMEM-F12, fetal bovine serum (FBS) and PenicillinStreptomycin (PS) antibiotic were obtained from Thermo Fisher Scientific. Chloroform, isopropanol, ethanol and ultrapure water were acquired from Sigma (St. Louis, $\mathrm{MO})$. 


\section{Umbilical cord tissue processing for cryopreservation}

Human UCTs are acquired from consenting cord blood and cord tissue donors who were screened against genetic and health history risks in accordance with FACT, AABB and FDA standards and approved by the Western Institutional Review Board (WIRB Protocol \#20002129). Briefly, human UCTs are collected in PBS-containing sterile collection cups with secured screw-cap lids (Corning). Upon delivery, UCT were quickly processed using multiple washes with sterile PBS containing $1 \%$ PS antibiotics $\mathrm{mix}(100 \mathrm{U} / \mathrm{ml}$ penicillin, $100 \mu \mathrm{g} / \mathrm{ml}$ streptomycin) at a Good Manufacturing/Tissue Practice facility. UCTs are sectioned into $0.5 \mathrm{~mm}$ squares, incubated in CryoStor CS10 at $4^{\circ} \mathrm{C}$, transferred to a controlled rate freezer and cryopreserved to $-90^{\circ} \mathrm{C}$. Final products are stored in the vapor phase of liquid nitrogen.

\section{MSC expansion of post-thaw umbilical cord tissue}

Frozen processed UCT samples are quickly thawed in a $37^{\circ} \mathrm{C}$ water bath and washed three times with sterile PBS. UCT samples were further sectioned into smaller pieces ( $\sim 2 \mathrm{~mm}^{2}$ pieces) and incubated with DMEM-F12 media. MSCs were expanded from the explanted UCT samples for 2 4 weeks with regular replenishment of DMEM-F12 media containing 10\% FBS every three days. MSC growth was visually confirmed by microscopy and after culture confluence of $>75 \%$, remaining UCT sections were removed from the dish. Adherent MSCs were washed once with sterile PBS and harvested with TrypLE solution (Life Technologies) and sub-cultured to flasks.

\section{MSC confirmation by flow cytometry}

Expanded MSCs were harvested using TrypLE solution. Cells were pelleted at $300 \times g$ for $5 \mathrm{~min}$, resuspended in $2 \mathrm{ml}$ of PBS through a $35 \mu \mathrm{m}$ nylon-mesh filter (Thermo Fisher Scientific), pelleted at $300 \times g$ for $5 \mathrm{~min}$ and resuspended to a final volume of $1 \mathrm{ml}$ of PBS. Filtered cells were dispensed to $100 \mu 1$ aliquots, incubated with mesenchymal stem cell marker CD73, CD90 and CD105 (Stemflow, Becton Dickinson) and shielded from light for $20 \mathrm{~min}$ at room temperature. Stained cells were reconstituted to a volume of $600 \mu 1$ with the addition of 500 $\mu 1$ of PBS prior to analysis by flow cytometry (FACSCalibur, Becton Dickinson) at $60 \mu \mathrm{l} / \mathrm{min}$ and 20,000 events.

\section{RNA extraction}

RNA from tissue was extracted using Trizol (Life Technologies) according to manufacturer's protocol. Briefly, $100 \mathrm{mg}$ of tissue were homogenized directly in 1 $\mathrm{ml}$ of Trizol reagent using a micro tissue disruptor (Kinematica, Bohemia, NY). For cell culture, RNA was harvested from confluent culture flasks using $1 \mathrm{ml}$ of Trizol after aspirating spent media, washing with ice-cold PBS and aspirating remaining liquid. Phase separation was achieved after the addition of $0.2 \mathrm{ml}$ of chloroform and centrifugation at $14,000 \times g$ for $15 \mathrm{~min}$ at $4^{\circ} \mathrm{C}$. Aqueous supernatant was transferred to a new microcentrifuge tube and RNA was precipitated with $0.5 \mathrm{ml}$ of isopropanol and centrifugation at $16,000 \times g$ for $10 \mathrm{~min}$ at $4^{\circ} \mathrm{C}$. Total RNA pellet was washed with $70 \%$ ethanol, allowed to air dry and resuspended in $50 \mu 1$ of ultrapure water. An aliquot of $1 \mu 1$ per sample was used for concentration determination by spectroscopy on a Nanodrop 2000 (Thermo Fisher Scientific) using absorbance reading at $260 \mathrm{~nm}$ and $280 \mathrm{~nm}$ wavelengths.

\section{cDNA conversion}

Total RNA samples were converted to cDNA using reverse transcriptase (iScript kit, Bio-Rad, Hercules, CA). Briefly, $2 \mu \mathrm{g}$ of total RNA samples were diluted to a reaction volume of $40 \mu 1$ containing $1 \mu 1$ of reverses transcriptase and $5 \mathrm{X}$ reaction buffer containing dNTP mixtures. Thermal cycling conditions were as follows: $25^{\circ} \mathrm{C}$ for $10 \mathrm{~min}, 42^{\circ} \mathrm{C}$ for $60 \mathrm{~min}$ and heat inactivation at $85^{\circ} \mathrm{C}$ for $10 \mathrm{~min}$.

\section{RT-PCR using SYBR green}

For RT-PCR, 50 ng of cDNA were used as template for screening of CD73, CD90, CD105, CDH-11 and GAPDH as internal control. Briefly, each reaction volume of $20 \mu 1$ contained $50 \mathrm{ng}$ of $\mathrm{cDNA}, 10 \mu \mathrm{mol} / \mathrm{L}$ of forward primer, $10 \mu \mathrm{mol} / \mathrm{L}$ of reverse primer, ultrapure water and $10 \mu 1$ of SYBR Green supermix (Bio-Rad). Human primer sequences used in this study are $\mathrm{CD} 73$ forward: CAGTACCAGGGCACTATCTGG, CD73 reverse: AGTGGCCCCTTTGCTTTAAT, CD90 forward: ATGAACCTGGCCATCAGCA, CD90 reverse: GTGTGCTCAGGCACCCC, CD105 forward: CCACTAGCCAGGTCTCGAAG, CD105 reverse: GATGCAGGAAGACACTGCTG, CDH-11 forward: GGTCTGGAACCAGTTCTTCG, CDH-11 reverse: TCTCGATCCAACGTCTTGGT, GAPDH forward: GAGTCAACGGATTTGGTCGT, and GAPDH reverse: TGGGATTTCCATTGATGACA. Reactions were amplified on a thermal cycler (CFX Connect, Bio-Rad) with thermal conditions set at: polymerase activation at $95^{\circ} \mathrm{C}$ for $3,40 \mathrm{am}$ plification cycles of $95^{\circ} \mathrm{C}$ for $15 \mathrm{sec}$ denaturating and $60^{\circ} \mathrm{C}$ for $60 \mathrm{sec}$ annealing, and melt curve analysis of $55 \sim 95^{\circ} \mathrm{C}$ at $0.5^{\circ} \mathrm{C}$ per $5 \mathrm{sec}$. Representative reactions were resolved on a $1.5 \%$ agarose gel at $90 \mathrm{~V}$ for $45 \mathrm{~min}$ and captured on 
by UV on an imager (Syngene PXi, Frederick, MD).

\section{Western blotting}

UCT samples of $1 \mathrm{~cm}$ were homogenized in RIPA lysis buffer (20 mM Tris- $\mathrm{HCl}$ ( $\mathrm{pH} 7.5$ ) containing $150 \mathrm{mM}$ $\mathrm{NaCl}, 1 \mathrm{mM}$ EDTA, $1 \mathrm{mM}$ EGTA, 1\% NP-40, 1\% sodium deoxycholate, $2.5 \mathrm{mM}$ sodium pyrophosphate, $1 \mathrm{mM} \beta$ -glycerophosphate, $1 \mathrm{mM}$ sodium vanadate and $0.002 \mathrm{mM}$ leupeptin) (Thermo Fisher Scientific) containing protease and phosphatase inhibitors (Thermo Fisher Scientific) using a micro tissue disruptor (Kinematica). For cell expansion, MSCs were cultured in $100-\mathrm{mm}^{2}$ dishes to $75 \sim$ $90 \%$ confluence. MSCs were washed 3 times with $5 \mathrm{ml}$ of cold PBS and harvested with $1 \mathrm{ml}$ of RIPA lysis buffer. Both tissue and cell homogenates were placed on ice for 10 minutes before centrifuging at $16,000 \times g$ for $10 \mathrm{~min}$ to pellet cellular debris. Supernatant was transferred to a sterile microcentrifuge tube and protein determination was performed on $2 \mu \mathrm{l}$ aliquots using the Bradford method (Bio-Rad). Protein lysates were diluted 1:1 (vol/vol) with $2 \mathrm{X}$ LDS buffer containing SDS (Life Technologies) and denatured at $95^{\circ} \mathrm{C}$ for $10 \mathrm{~min}$. Protein extracts were subjected to a variable $4 \sim 12 \%$ SDS-polyacrylamide gel electrophoresis (NuPAGE Novex Bis-Tris Gels, Life Technologies) for $45 \mathrm{~min}$ at $200 \mathrm{~V}$, and transferred onto a nitrocellulose membrane for $75 \mathrm{~min}$ at $100 \mathrm{~V}$. The membrane was washed with Tris buffered saline (TBS, Sigma), blocked with 5\% dried nonfat milk (Bio-Rad) and 5\% BSA (Sigma) in 1\% tween-TBS and probed with antibody against CDH-11 (1:1,000) or GAPDH $(1: 2,500)$ as a visual loading control. Primary antibodies were followed by secondary antibody IgG linked to horseradish peroxidase conjugate $(1: 2,500)$. The blots were visualized by enhanced chemiluminescence (Thermo Fisher Scientific) and scanned using the PXi imager (Syngene).

\section{Statistical analyses}

Data are expressed as mean $\pm S D$ from $n \geq 3$ experiments. Mean comparisons were performed with two-way ANOVA using SigmaPlot (Systat Software, San Jose, CA). Statistical significance was set at $\mathrm{p}<0.05$.

\section{Results}

\section{Fresh and cryopreserved UCT expresses CD73, CD 90 and CD105 mRNA}

Donated umbilical cord tissues were manually dissected for cord lining and Wharton's jelly, excluding blood vessels. UCTs were de-identified and assigned 7-digit local identification numbers (3548618, 3548627, 3548629 and
3548920). Umbilical cords were thoroughly rinsed in PBS to remove blood vessels and blood components and section into $1-\mathrm{cm}^{3}$ pieces. One set of $1-\mathrm{cm}^{3}$ sections were processed for RNA and protein while remaining sections were frozen in CryoStor CS10. Fresh sample sections were screened for CD73, CD90 and CD105 by RT-PCR shown in Fig. 1A. Despite the individual sample variability, the findings show that CD73 mRNA expression averaged 0.123 \pm 0.019 fold, CD90 mRNA expression averaged $0.038 \pm 0.008$-fold and CD105 mRNA expression averaged 0.045 \pm 0.009 -fold. When the same frozen samples were thawed and processed for RNA after 30 weeks in the vapor phase of liquid nitrogen storage, the presence of CD73, CD90 and CD105 mRNA expression by SYBR green remained detectable as shown in Fig. 1B. Similar to the fresh UCT sample sections, the frozen sections varied individually for CD73 mRNA expression at $0.064 \pm 0.008$-fold, CD90 mRNA expression at $0.035 \pm 0.005$-fold and CD105 mRNA expression at $0.050 \pm 0.010$-fold. While the data for averaged CD73 mRNA expression shows a statistically significant loss with $\mathrm{p}<0.05$ for frozen samples as compared to fresh samples, differences in averaged CD90 and CD105 mRNA expression for frozen samples compared to fresh samples were not detected, rendering an inconclusive decision on whether long-term storage in the vapor phase of liquid nitrogen contributes to deterioration of tissue samples. This experiment was repeated with four different donors (coded as 3563430, 3563674, 3563695 and 3563803) in Supplementary Fig. S1 showing CD73, CD90 and CD105 mRNA expression from fresh (Fig. S1A) and frozen UCT (Fig. S1B). Signal loss in frozen UCT was not observed for CD73 and CD90 but was observed for CD105 at $34.7 \pm 17.7 \%$. This signal loss was not statistically significant for this set of donors. Combined CD73, CD90 and CD105 mRNA expression for UCT from all donors did not correlate with tissue deterioration.

\section{Fresh and cryopreserved UCT expresses CDH-11 mRNA}

Because CDH-11 is predominantly expressed in mesenchymal stem cells (17), it was postulated that CDH-11 could serve as a quick screening marker for the presence of MSC in the fresh and frozen samples. Screening of fresh and cryopreserved UCT sections for CDH-11 by RT-PCR shows that CDH-11 can be detected in fresh samples (Fig. 2A) as well as cryopreserved samples stored for 30 weeks (Fig. 2B). The results showed that CDH-11 mRNA expression averaged $0.007 \pm 0.003$-fold for fresh samples whereas CDH-11 mRNA expression averaged $0.004 \pm 0.002$-fold for frozen samples in storage for 30 weeks. Average loss for the combined cord tissue samples 
A
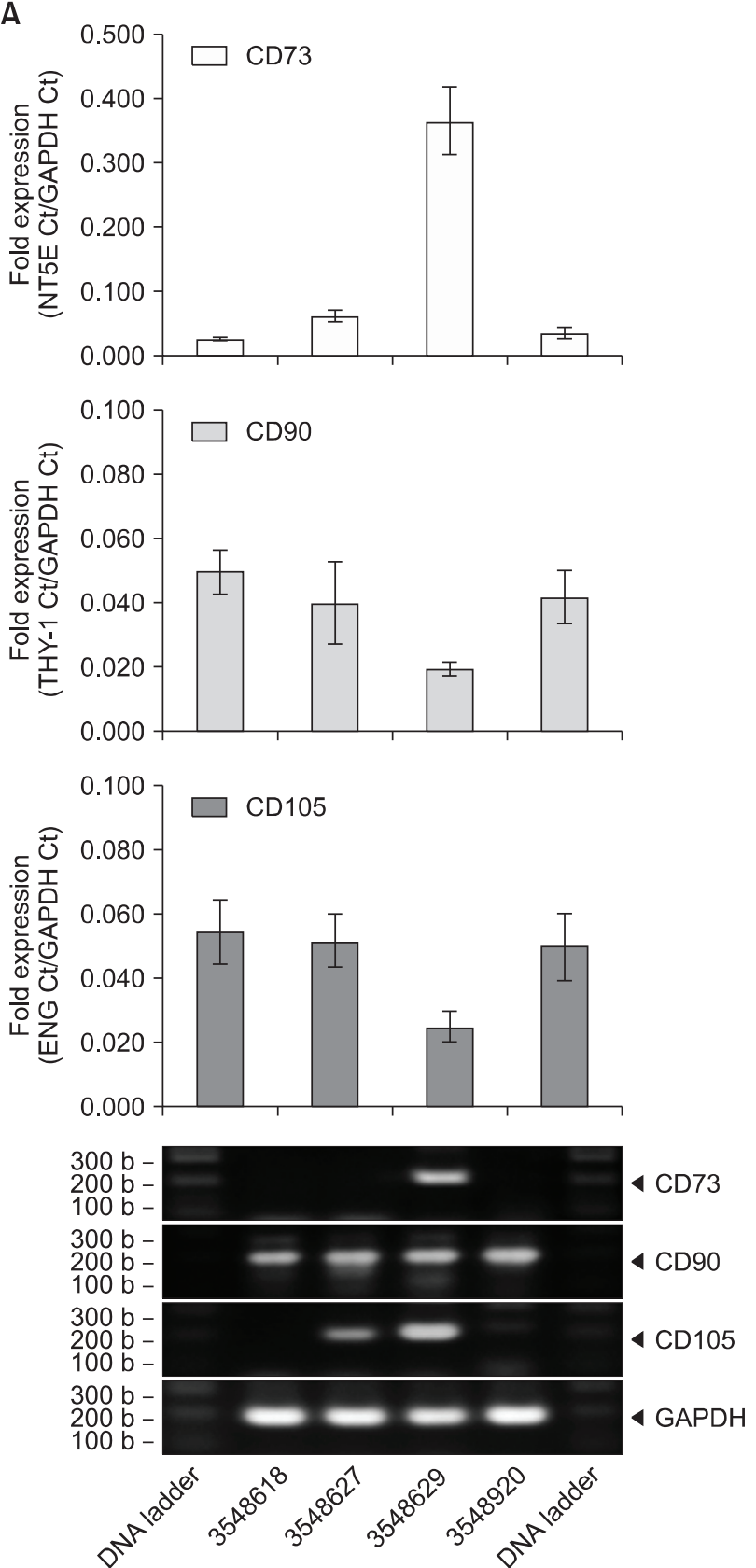

B
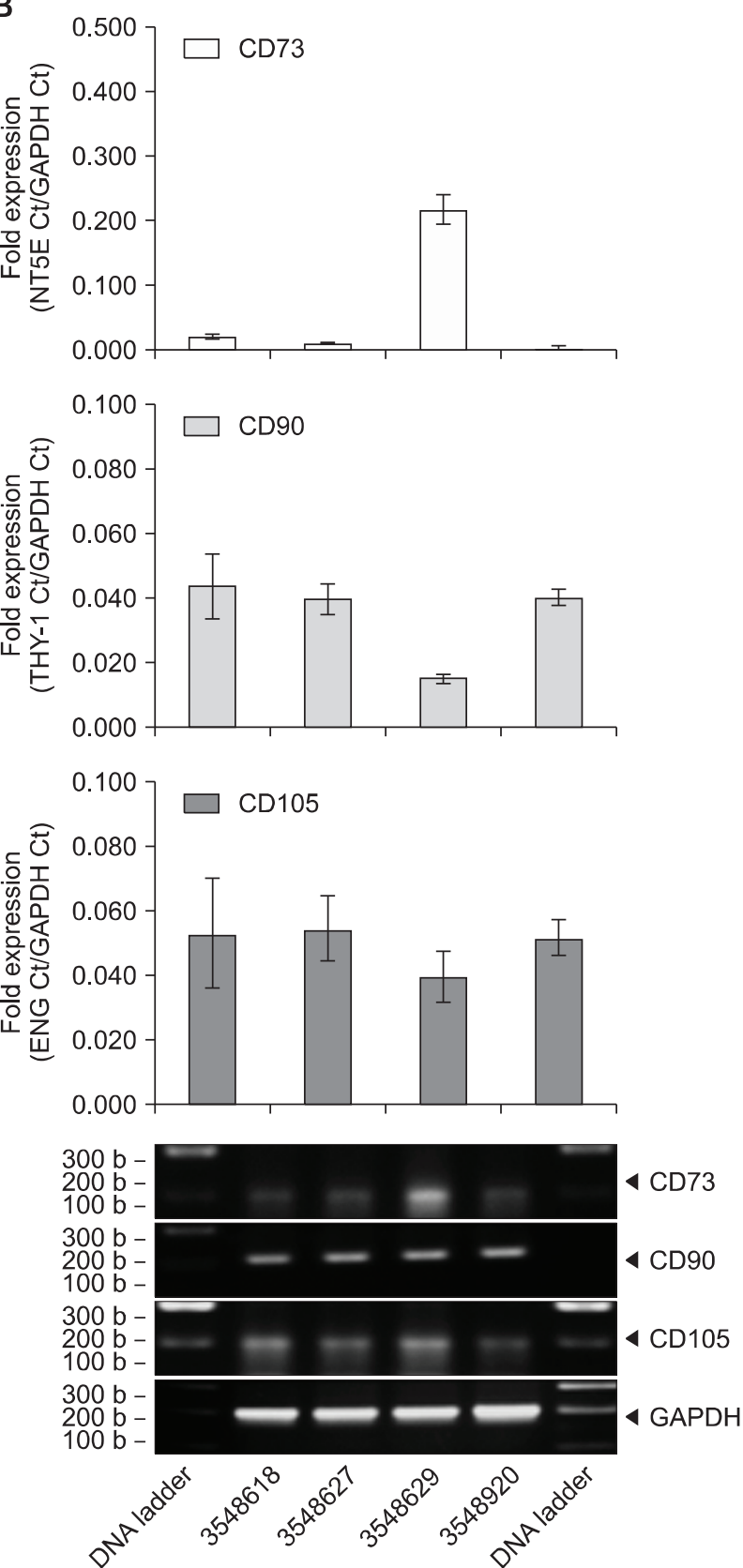

Fig. 1. Fresh and Cryopreserved UCT expresses CD73, CD90 and CD105 mRNA. (A) Fresh and (B) cryopreserved UCT from 4 different donors were processed for RNA, converted to CDNA and screened for CD73, CD90 and CD105 by RT-PCR. GAPDH was used as an internal control and data are expressed as mean fold expression against $\mathrm{GAPDH} \pm \mathrm{SD}$ from 3 experiments. Representative reactions were resolved on a $1.5 \%$ agarose gel and captured on an imager.

were $43.8 \pm 19.1 \%$; however, sample size tested rendered the data statistically inconclusive on correlating CDH-11 mRNA stability to MSC stability in the cryopreservation process. This experiment was repeated with 4 different donors showing CDH-11 mRNA expression loss at 14.3 \pm $21.9 \%$ in frozen UCT compared to fresh UCT (Fig. S3A and S3B), but was not statistically significant to determine tissue instability in long term storage.

\section{MSC expansion from UCT expresses CD73, CD90 and CD105}

The isolation and recoverability of MSCs from UCT was demonstrated using cord tissues from different donors. Fresh UCT sections were processed for MSC isolation and 
A

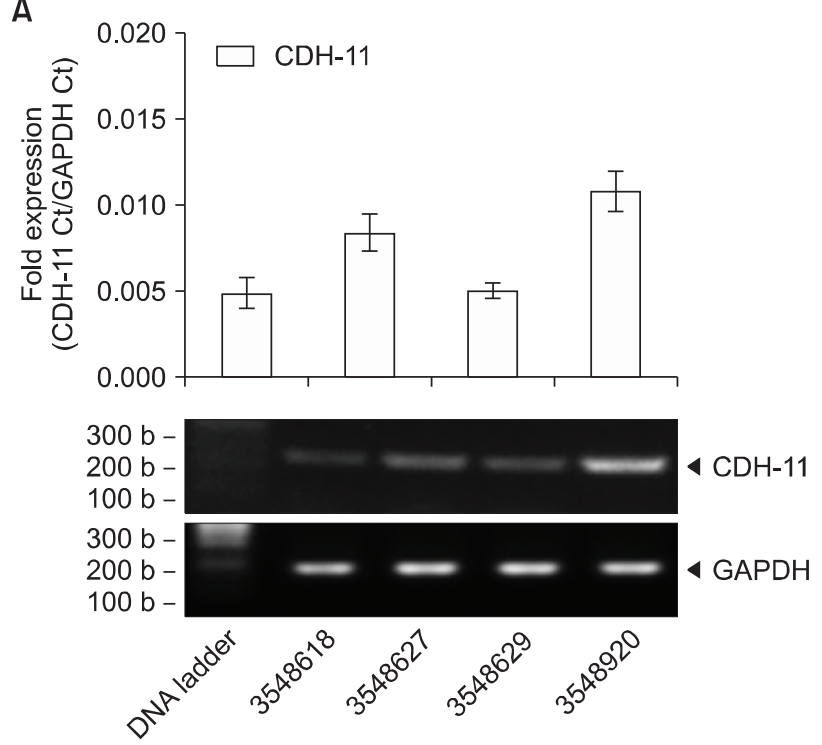

B

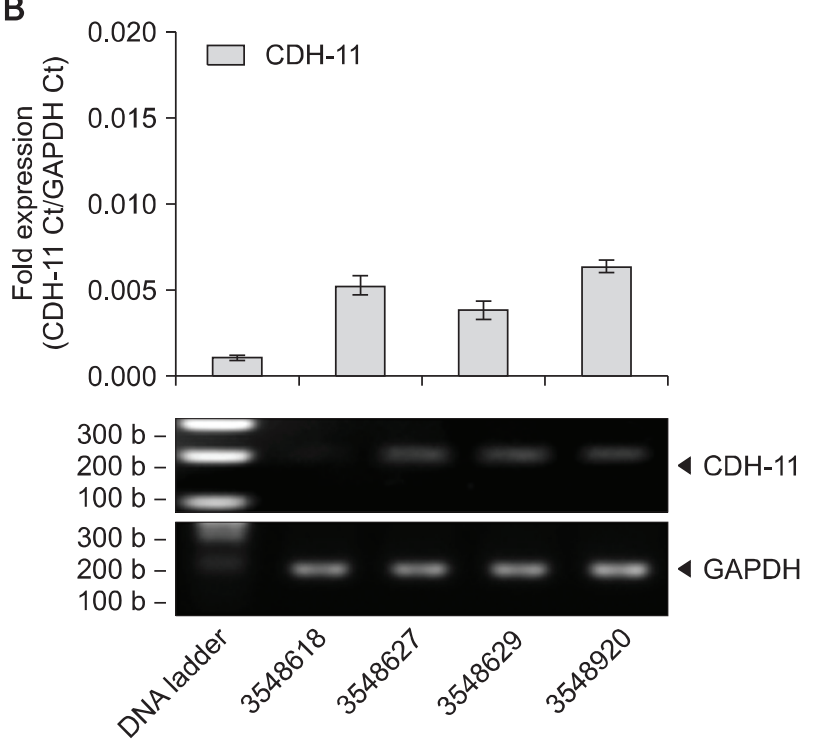

Fig. 2. Fresh and cryopreserved UCT expresses $\mathrm{CDH}-11$ mRNA. (A) Fresh and (B) frozen UCT from 4 different donors were processed for RNA, converted to CDNA and screened for CDH-11 by RT-PCR. GAPDH was used as an internal control and data are expressed as mean fold expression against $\mathrm{GAPDH} \pm \mathrm{SD}$ from 3 experiments. Representative reactions were resolved on a 1.5\% agarose gel and captured on an imager.

expansion in vitro. Expanded MSCs were allowed to propagate to increase cell counts in culture flasks shown in Fig. 3. Morphological analysis shows that these MSCs exhibit an adherent, fibroblastic characteristic and that these MSC cultures were devoid of hematopoietic contamination. UCT tissue pieces were cultured in petri dishes for $2 \sim 3$ weeks to allow MSCs (Passage 0, P0) to migrate and expand with replenished media every 3 days. After 75\% confluency of MSC population, cultures are further propagated into subsequent flasks as P1 and P2. MSC cultures (P1 or P2) were processed for RNA, converted to cDNA and screened for CD90, CD73 and CD105 mRNA by RT-PCR shown in Fig. 4A. CD73 mRNA expression averaged $0.049 \pm 0.002$-fold for sample 3551858 and $0.071 \pm$ 0.001-fold for sample 3551710. CD90 mRNA expression averaged $0.173 \pm 0.008$-fold for sample 3551858 and $0.096 \pm$ 0.003-fold for sample 3551710. CD105 mRNA expression averaged $0.005 \pm 0.001$-fold for sample 3551858 and $0.007 \pm 0.001$-fold for sample 3551710. MSCs expanded from cryopreserved UCT samples for 30 weeks in the vapor phase of liquid nitrogen presented similar mRNA expression for CD73, CD90 and CD105 by RT-PCR shown in Fig. 4B. CD73 mRNA expression averaged 0.074 \pm 0.005 -fold for sample 3551710 and $0.181 \pm 0.006$-fold for sample 3556064. CD90 mRNA expression averaged $0.083 \pm 0.008$-fold for sample 3551710 and $0.205 \pm 0.005$-fold for sample 3556064. CD105 mRNA expression averaged $0.006 \pm 0.001$-fold for sample 3551710 and $0.011 \pm 0.001$-fold for sample 3551710. A repeat of this experiment with four different donors showed a signal loss in CD73 expression by $34.7 \pm 12.6 \%$ whereas no statistical loss was observed for CD90 and CD105 (Supplementary Fig. S2A and S2B).

\section{MSC expansion expresses CD73, CD90 and CD105 surface markers}

MSC culture expansions from both fresh and cryopreserved UCT were confirmed by flow cytometry for the surface expression of CD73, CD90 and CD105 shown in Fig. 5A. An additional positive MSC marker CD44 was used in the representative flow cytometric output. Negative controls consisting of fluorescence conjugated secondary antibodies were used to demonstrate specificity shown in Fig. 5B. The absence of CD34 and CD45 expression were used as additional markers of MSC profiling (18, 19). Both MSC expansions from fresh and cryopreserved UCT samples showed characteristic surface expression of CD73, CD90 and CD105 and lacked CD34 and CD45 expression (data not shown). MSC expansion profiles averaged 99.8 $\pm 0.4 \%$ CD73 positive, $99.8 \pm 0.2 \%$ CD90 positive and $97.4 \pm 2.2 \%$ CD105 positive by flow cytometry. Additionally, MSC were $98.8 \pm 1.0 \%$ positive for CD44. In vitro expansion of MSCs were $92.8 \pm 2.3 \%$ viable using $7-\mathrm{AAD}$ as summarized in Fig. 5C. These data show that in vitro expansion can generate a highly enriched population of MSC as characterized by flow cytometry. This experiment was repeated with four different donors that showed sim- 


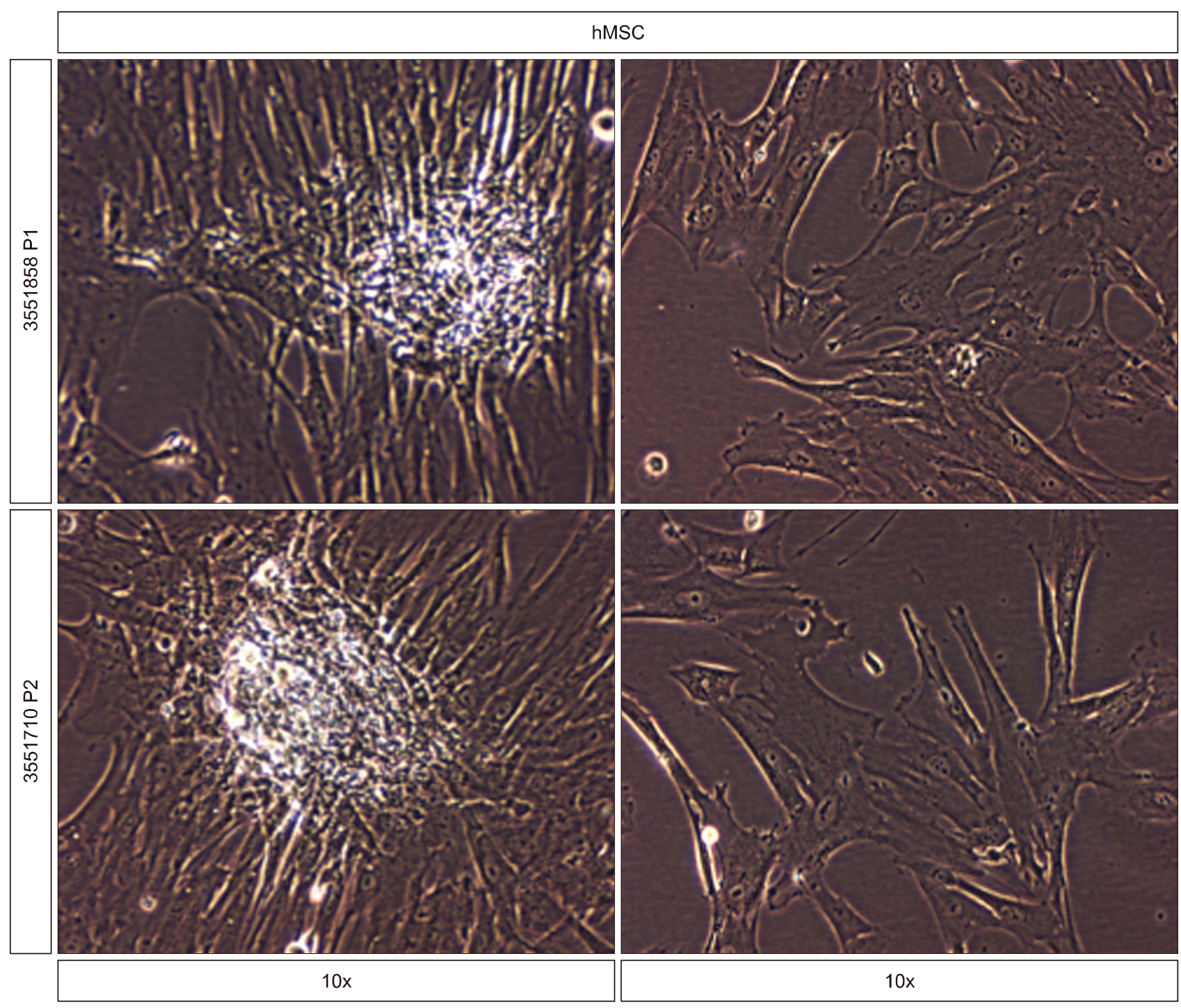

Fig. 3. Representative microscopy of expanded MSC from cryopreserved UCT. MSC expansion cultures were propagated from cryopreserved UCT. Representative phase-contrast field of views were captured on a digital microscope.

ilar flow cytometric prolife averaging 95 99\% positive surface markers CD73, CD90, CD105 and CD44 (data not shown).

\section{MSC expansion from UCT expresses CDH-11}

MSC expansions from fresh UCT tissue pieces were screened for $\mathrm{CDH}-11$ expression by RT-PCR shown in Fig. 6A. Expression of CDH-11 mRNA expression averaged $0.198 \pm 0.011$-fold for sample 3551858 and $0.121 \pm$ 0.008-fold for sample 3551710. Similarly, MSCs expanded from cryopreserved UCT samples were screened for CDH-11 mRNA expression shown in Fig. 6B. CDH-11 mRNA expression averaged $0.107 \pm 0.007$-fold for sample 3551858 and $0.088 \pm 0.004$-fold for sample 3551710 . A re- peat of this experiment with four different donors did not show statistically significant CDH-11 mRNA loss in MSC expansion from frozen UCT compared to fresh UCT (Supplementary Fig. S3C and S3D).

To show that CDH-11 mRNA expression does correlate to protein expression, MSCs were harvested and processed for protein. Western blotting using CDH-11 antibody shows MSC expansions express the $\mathrm{CDH}-11$ surface marker for mesenchymal profile shown in Fig. 6C. This set of experiments provides evidence that $\mathrm{CDH}-11$ could be used as a quick and simple marker of mesenchymal lineage to verify the presence of MSC in UCT tissue. Similarly, fresh and frozen UCT from four different donors expressed CDH-11 protein shown in Supplementary Fig. S4A and 
A
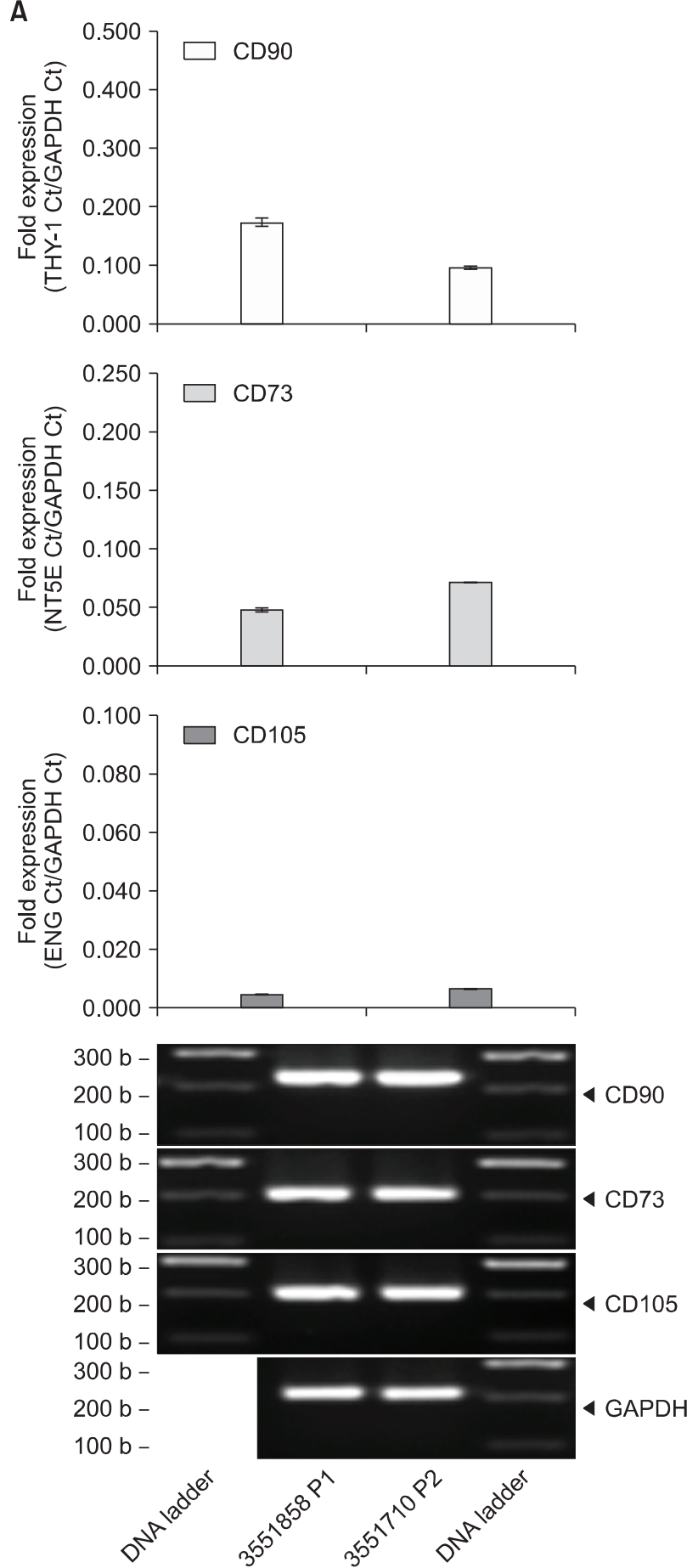

B
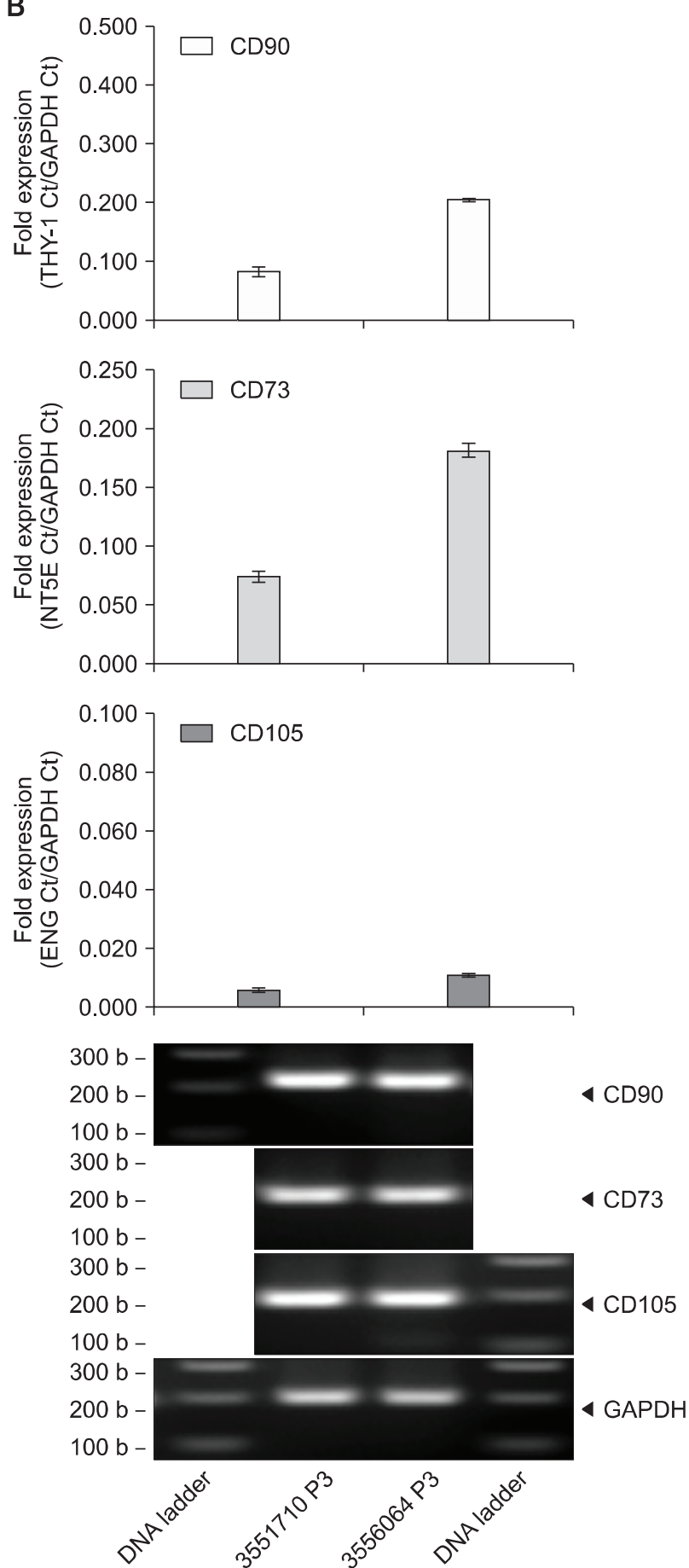

Fig. 4. MSC expansion from fresh and cryopreserved UCT express CD90, CD73 and CD105 mRNA. MSC expanded from (A) fresh and (B) cryopreserved UCT from 2 different donors were processed for RNA, converted to CDNA and screened for CD73, CD90 and CD105 by RT-PCR. GAPDH was used as an internal control and data are expressed as mean fold expression against GAPDH \pm SD from 3 experiments. Representative reactions were resolved on a 1.5\% agarose gel and captured on an imager.

S4B, respectively. MSC expanded from fresh and frozen UCT expressed CDH-11 protein shown in Supplementary Fig. S4C and S4D, respectively, and correlated with
CDH-11 mRNA expression. 

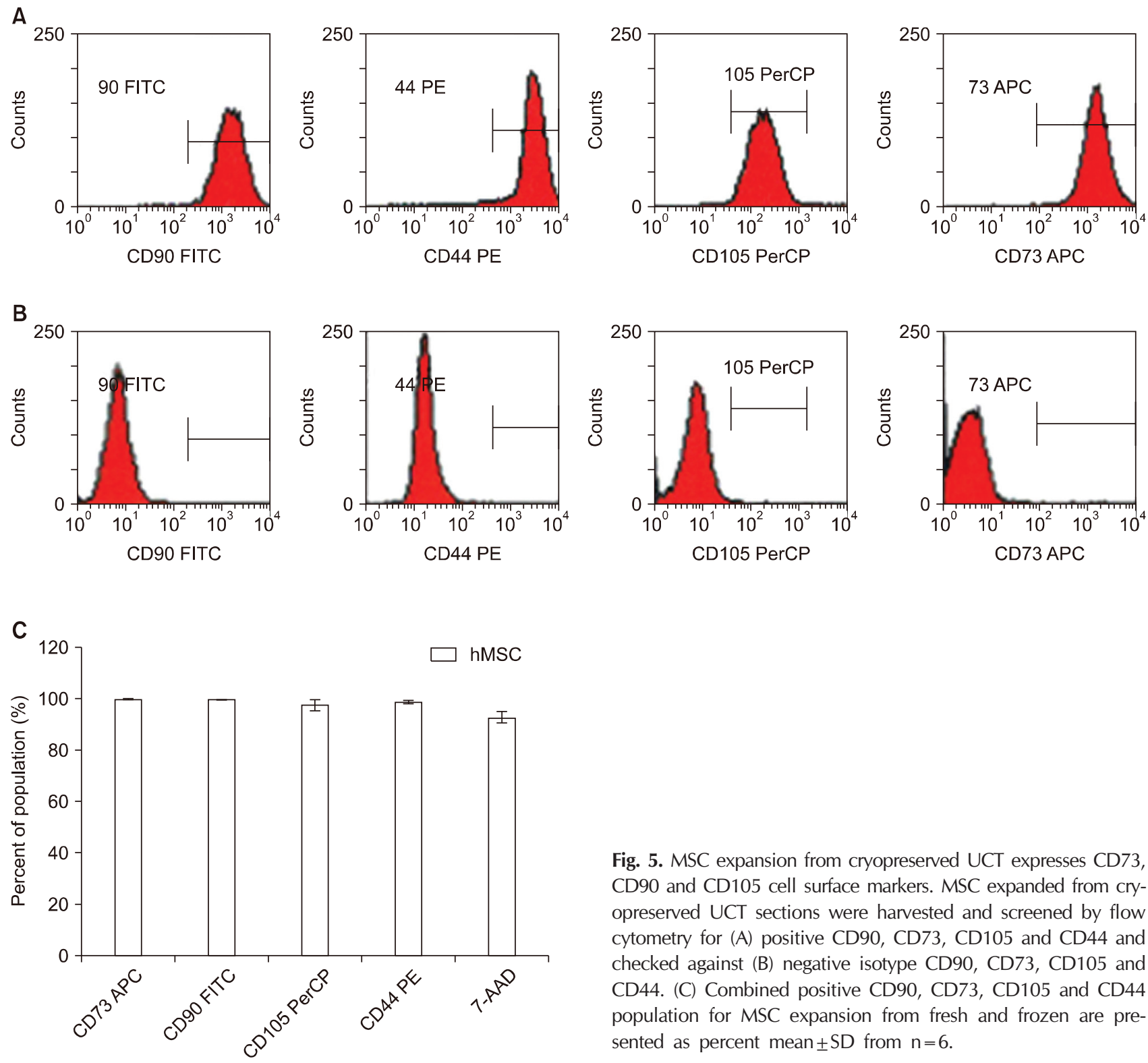

\section{Discussion}

Overall, this study demonstrated that UCT processed and cryopreserved in 10\% DMSO retains detectable MSC markers when stored in the vapor phase of liquid nitrogen. The potential of cryopreserved UCT to generate viable MSCs were achieved by RT-PCR detection of MSC markers without the commitment of in vitro expansion for MSCs. However, to confirm the validity of the RT-PCR results, MSC were expanded from post-thawed UCT and characterized by flow cytometry for MSC markers as outlined in the industry guideline set by the International Society for Cellular Therapy (12). Indeed, transcriptome

Fig. 5. MSC expansion from cryopreserved UCT expresses CD73, CD90 and CD105 cell surface markers. MSC expanded from cryopreserved UCT sections were harvested and screened by flow cytometry for (A) positive CD90, CD73, CD105 and CD44 and checked against (B) negative isotype CD90, CD73, CD105 and CD44. (C) Combined positive CD90, CD73, CD105 and CD44 population for MSC expansion from fresh and frozen are presented as percent mean \pm SD from $n=6$.

screening has been proposed for detecting, characterizing and identifying umbilical cord stem cells to determine the suitability for clinical application but has not been adapted for screening whole umbilical cord sections (20). The method of RT-PCR or PCR screening for MSC markers can be performed within hours whereas commitment to in vitro expansion requires $3 \sim 6$ weeks to generate adequate cell numbers for flow cytometric analysis. Post-thawed cryopreserved UCT sections were successfully expanded for MSC in vitro and expressed MSC markers in a similar pattern identified by flow cytometry.

To accelerate the screening process for MSC stability in the cryopreserved UCTs, a singular target $\mathrm{CDH}-11$ was 
A

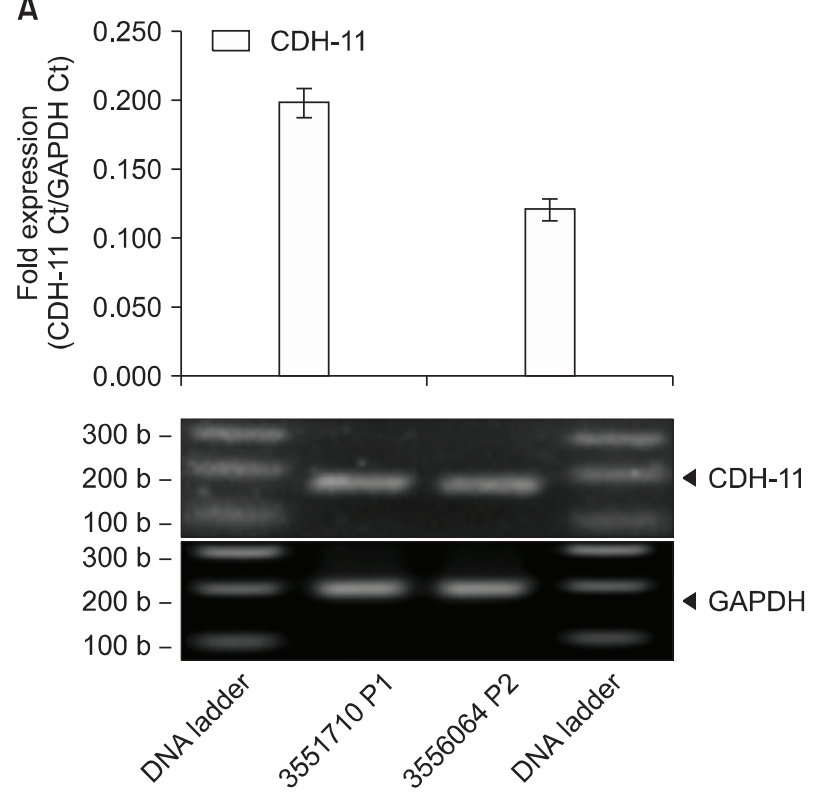

C

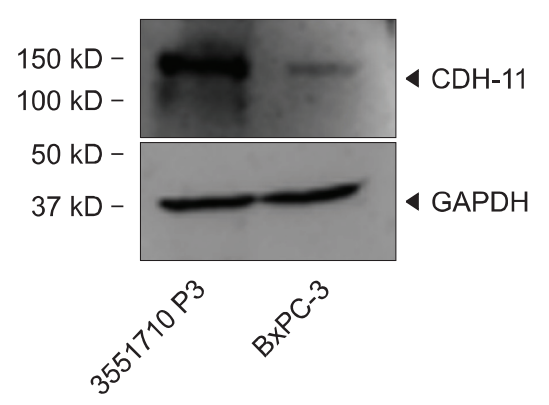

B

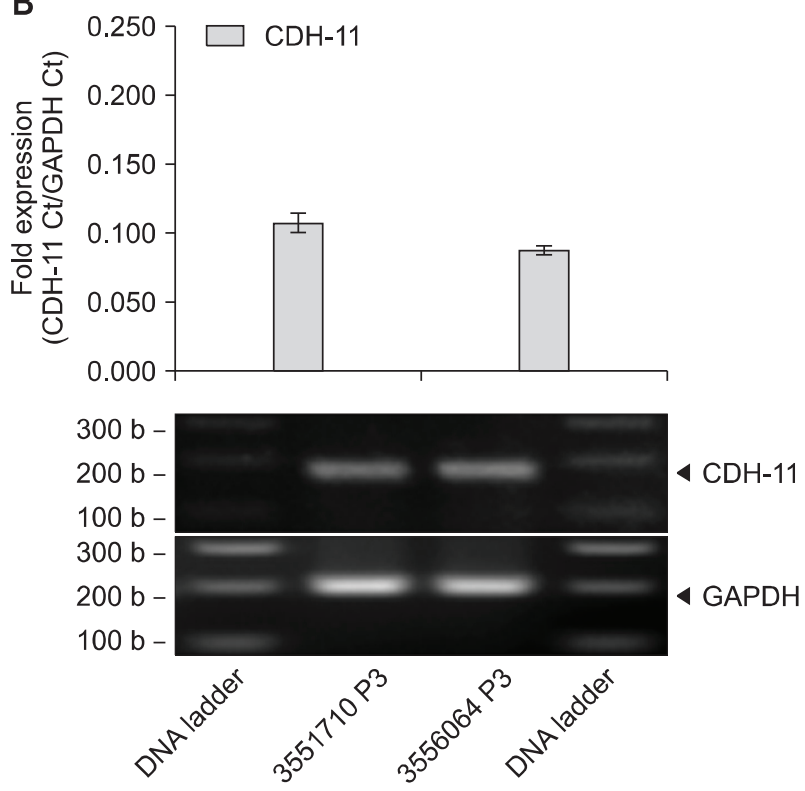

Fig. 6. MSC expansion from cryopreserved UCT expresses CDH-11. MSCs expanded from (A) fresh and (B) cryopreserved UCT sections were processed for RNA, converted to CDNA and screened for CDH-11 by RT-PCR. GAPDH was used as an internal control and data are expressed as mean fold expression against $\mathrm{GAPDH}_{ \pm} \mathrm{SD}$ from 3 experiments. Representative reactions were resolved on a $1.5 \%$ agarose gel and captured on an imager. (C) MSCs were processed for protein, denatured in loading buffer containing SDS, resolved on a $4 \sim 20 \%$ pre-cast gel and transferred onto nitrocellulose membranes. Membranes were probed with $\mathrm{CDH}-11$ or GAPDH antibodies, visualized by $\mathrm{ECL}$ and captured on an imager.

used to distinguish MSC from potential HSC contamination. In addition to adhesion and migration, $\mathrm{CDH}-11$ has been reported to mediate stem cell differentiation (21). MSCs were shown to differentiate to osteocytes and chondrocytes through CDH-11 (22). The result showed that CD73, CD90 and CD105 as well as CDH-11 are detectable by RT-PCR and qPCR from fresh and cryopreserved UCT. Our results showed that RT-PCR monitoring of cryopreserved cord tissue quality after long-term vapor phase of liquid nitrogen storage provided a quick and feasible assessment for stability. This method can be employed to quickly identify the presence of MSC in cryopreserved UCT without the time commitment of isolating and expanding potentially non-viable tissue. The minimal mark- ers that define MSCs include the expression of CD73, CD90, CD105, CD44 and the absence of CD34 and CD45 expression by flow cytometry as the accepted industry standard (12). However, other MSC markers have been reported to include CD26, Stro-1, SSEA-4, CD271, and CD146 (23). Our results showed that MSC marker CDH-11 could be detected by RT-PCR as well as Western blotting from expanded cultures. The results suggest that $\mathrm{CDH}-11$ may serve as an alternative or additional MSC marker. $\mathrm{CDH}-11$ is a constituent of the cadherin family of cell-cell adhesion protein that also mediates cell migration (24). Because CDH-11 is expressed on the cell surface, this protein can be employed for screening by flow cytometry when conjugated to a fluorescent antibody 
against CDH-11. Whether as a single target for MSC detection or combined with CD73, CD90 and CD105, CDH-11 can serve as a viable RT-PCR marker for a quick quality assessment of cryopreserved UCT.

While this study did not elaborate extensively on MSC expansion methodology, propagating in vitro MSCs is dependent upon several factors that include but not limited to isolation technique and media supplementation. It is unclear whether the isolation methodologies may compromise MSC expansion and characterization of surface markers. Several groups have outlined MSC isolation methodology for in vitro expansion, particularly noting an abundance of MSCs in Wharton's Jelly (25). MSCs have been successfully isolated from endometrial decidual tissue and menstrual blood (26) and amniotic membrane tissue (27); however, the abundant source of MSCs appears in the Wharton's Jelly of the UCT (28). MSC from the entire cord have also been reported and whole cord tissue or Wharton's Jelly MSC isolation yielded comparable MSCs quality using the identical isolation technique (29). Isolation of MSC by conventional enzyme digestion and tissue explant has been shown to produce viable MSC (30). Enzymatic digestion of UCT using collagenase, hyaluronidase and trypsin without mechanical dispersion resulted in viable MSC prior to cryopreservation and post thaw (31). Furthermore, these expanded MSCs can also be characterized by RT-PCR for MSC markers CD73, CD90 and CD105. A majority of MSCs were reportedly derived from Wharton's Jelly and to a lesser extent, from cord tissue lining $(18,19,25)$. For this study, the entire cord tissue after removal of residual blood components was cryopreserved to maintain the potential for other stem cell types to be isolated for future use.

To ensure isolated MSCs can be clinically utilized, several groups have attempted to propagate these cells using xenobiotic-free reagents to minimize potential immune responses in vivo (32-34). The substitution of human umbilical cord blood plasma for animal serum in the culturing of MSCs has resulted with success (32). Human umbilical cord blood plasma used as supplement supported ex vivo MSC growth as a replacement for FBS (35). Similarly, platelet lysate as well as activated platelet lysate substitution of fetal bovine serum produced viable MSCs (36). MSCs propagated from Wharton Jelly's in commercial xenobiotic-free medium without serum were confirmed by cell surface expression of CD44, CD73, CD90 and CD105 markers (33). Other groups utilizing the xenobiotic- and serum-free media have shown this condition can generate viable MSC in vitro and, thus, minimize potential immunizing effects when used clinically $(37,38)$.
Umbilical cord extract used for serum and combined with collagen purified from umbilical cord for extracellular matrix coating resulted in viable in vitro expansion of MSCs as an alternative to animal serum and extracellular matrix coating of culture plates (34).

One report showed that long-term culturing in serum-free, xenobiotic-free medium of expanded MSC passed 15 times did not exhibit aberrant transformation (39). This was also supported by another report that culturing of MSCs up to 15 passages did not alter positive MSC marker expression for CD44, CD90, and CD105 (40). For this study, MSC expansions were tested up to 5 passages that retained CD73, CD90, CD105 and CDH-11 expression by flow cytometry and RT-PCR (data not shown). We showed that cryopreserved umbilical cord tissue test positive for MSC markers CD73, CD90, CD105 and $\mathrm{CDH}-11$ by RT-PCR. Additionally, MSC expanded from sections of post-thawed UCT in vitro expressed CD73, CD90, and CD105 by RT-PCR and flow cytometry. We also showed that these in vitro expanded MSCs also express CDH-11 by RT-PCR and Western blotting. Quick analysis for the presence of MSCs in cryopreserved UCT sections after long-term liquid nitrogen vapor phase storage can be achieved using RT-PCR for current MSC markers or alternatively, a CDH-11 RT-PCR screen may satisfy this requirement.

\section{Acknowledgements}

H.P. performed experiments, analyzed the data and wrote the manuscript. R.T., C.B. and M.C. performed experiments. H.P., R.T., M.W. and M.C. developed experimental design and interpreted the data. All read and finalized the manuscript.

\section{Potential Conflict of Interest}

The authors have no conflicting financial interest.

\section{Supplementary Materials}

Supplementary data including four figures can be found with this article online at http://pdf.medrang.co.kr/paper/ pdf/IJSC/IJSC-11-s17-015.pdf.

\section{References}

1. Weiner LP. Definitions and criteria for stem cells. Methods Mol Biol 2008;438:3-8

2. Trounson A, McDonald C. Stem cell therapies in clinical trials: progress and challenges. Cell Stem Cell 2015;17: $11-22$ 
3. Thomas ED. Bone marrow transplantation: a review. Semin Hematol 1999;36(4 Suppl 7):95-103

4. Reinke S, Dienelt A, Blankenstein A, Duda GN, Geissler S. Qualifying stem cell sources: how to overcome potential pitfalls in regenerative medicine? J Tissue Eng Regen Med 2016;10:3-10

5. Friedenstein AJ, Petrakova KV, Kurolesova AI, Frolova GP. Heterotopic of bone marrow. Analysis of precursor cells for osteogenic and hematopoietic tissues. Transplantation 1968;6:230-247

6. Pittenger MF, Mackay AM, Beck SC, Jaiswal RK, Douglas R, Mosca JD, Moorman MA, Simonetti DW, Craig S, Marshak DR. Multilineage potential of adult human mesenchymal stem cells. Science 1999;284:143-147

7. Jadalannagari S, Aljitawi OS. Ectodermal differentiation of Wharton's Jelly mesenchymal stem cells for tissue engineering and regenerative medicine applications. Tissue Eng Part B Rev 2015;21:314-322

8. Abumaree M, Al Jumah M, Pace RA, Kalionis B. Immunosuppressive properties of mesenchymal stem cells. Stem Cell Rev 2012;8:375-392

9. Nagamura-Inoue T, He H. Umbilical cord-derived mesenchymal stem cells: Their advantages and potential clinical utility. World J Stem Cells 2014;6:195-202

10. Fazzina R, Mariotti A, Procoli A, Fioravanti D, Iudicone P, Scambia G, Pierelli L, Bonanno G. A new standardized clinical-grade protocol for banking human umbilical cord tissue cells. Transfusion 2015;55:2864-2873

11. Van Pham P, Phan NK. Production of good manufacturing practice-grade human umbilical cord blood-derived mesenchymal stem cells for therapeutic use. Methods Mol Biol 2015;1283:73-85

12. Dominici M, Le Blanc K, Mueller I, Slaper-Cortenbach I, Marini F, Krause D, Deans R, Keating A, Prockop Dj, Horwitz E. Minimal criteria for defining multipotent mesenchymal stromal cells. The International Society for Cellular Therapy position statement. Cytotherapy 2006;8: 315-317

13. Maleki M, Ghanbarvand F, Reza Behvarz M, Ejtemaei M, Ghadirkhomi E. Comparison of mesenchymal stem cell markers in multiple human adult stem cells. Int J Stem Cells 2014;7:118-126

14. Chatzistamatiou TK, Papassavas AC, Michalopoulos E, Gamaloutsos C, Mallis P, Gontika I, Panagouli E, Koussoulakos SL, Stavropoulos-Giokas C. Optimizing isolation culture and freezing methods to preserve Wharton's jelly's mesenchymal stem cell (MSC) properties: an MSC banking protocol validation for the Hellenic Cord Blood Bank. Transfusion 2014;54:3108-3120

15. Simonneau L, Kitagawa M, Suzuki S, Thiery JP. Cadherin 11 expression marks the mesenchymal phenotype: towards new functions for cadherins? Cell Adhes Commun 1995; 3:115-130

16. Christopoulos PF, Bournia VK, Panopoulos S, Vaiopoulos A, Koutsilieris M, Sfikakis PP. Increased messenger RNA levels of the mesenchymal cadherin-11 in the peripheral blood of systemic sclerosis patients correlate with diffuse skin involvement. Clin Exp Rheumatol 2015;33(4 Suppl 91):S36-S39.

17. MacCalman CD, Furth EE, Omigbodun A, Bronner M, Coutifaris C, Strauss JF 3rd. Regulated expression of cadherin-11 in human epithelial cells: a role for cadherin-11 in trophoblast-endometrium interactions? Dev Dyn 1996; 206:201-211

18. Shi S, Jia S, Liu J, Chen G. Accelerated regeneration of skin injury by co-transplantation of mesenchymal stem cells from Wharton's jelly of the human umbilical cord mixed with microparticles. Cell Biochem Biophys 2015; 71:951-956

19. Roy S, Arora S, Kumari P, Ta M. A simple and serum-free protocol for cryopreservation of human umbilical cord as source of Wharton's jelly mesenchymal stem cells. Cryobiology 2014;68:467-472

20. Mechiche Alami S, Velard F, Draux F, Siu Paredes F, Josse J, Lemaire F, Gangloff SC, Graesslin O, Laurent-Maquin D, Kerdjoudj H. Gene screening of Wharton's jelly derived stem cells. Biomed Mater Eng 2014;24(1 Suppl):53-61

21. Alimperti S, Andreadis ST. CDH2 and CDH11 act as regulators of stem cell fate decisions. Stem Cell Res 2015;14: 270-282

22. Kii I, Amizuka N, Shimomura J, Saga Y, Kudo A. Cell-cell interaction mediated by cadherin-11 directly regulates the differentiation of mesenchymal cells into the cells of the osteo-lineage and the chondro-lineage. J Bone Miner Res 2004;19:1840-1849

23. Lv FJ, Tuan RS, Cheung KM, Leung VY. Concise review: the surface markers and identity of human mesenchymal stem cells. Stem Cells 2014;32:1408-1419

24. Boscher C, Mège RM. Cadherin-11 interacts with the FGF receptor and induces neurite outgrowth through associated downstream signalling. Cell Signal 2008;20:1061-1072

25. Bongso A, Fong CY. The therapeutic potential, challenges and future clinical directions of stem cells from the Wharton's jelly of the human umbilical cord. Stem Cell Rev 2013;9:226-240

26. Rossignoli F, Caselli A, Grisendi G, Piccinno S, Burns JS, Murgia A, Veronesi E, Loschi P, Masini C, Conte P, Paolucci P, Horwiz EM, Dominici M. Isolation, characterization, and transduction of endometrial decidual tissue multipotent mesenchymal stromal/stem cells from menstrual blood. Biomed Res Int 2013 doi: 10.1155/2013/901821

27. Pirjali T, Azarpira N, Ayatollahi M, Aghdaie $\mathrm{MH}$, Geramizadeh B, Talai T. Isolation and characterization of human mesenchymal stem cells derived from human umbilical cord Wharton's Jelly and amniotic membrane. Int J Organ Transplant Med 2013;4:111-116

28. Harris DT. Umbilical cord tissue mesenchymal stem cells: characterization and clinical applications. Curr Stem Cell Res Ther 2013;8:394-399

29. Hendijani F, Sadeghi-Aliabadi H, Haghjooy Javanmard S. Comparison of human mesenchymal stem cells isolated by explant culture method from entire umbilical cord and 
Wharton's jelly matrix. Cell Tissue Bank 2014;15:555-565 30. Han YF, Tao R, Sun TJ, Chai JK, Xu G, Liu J. Optimization of human umbilical cord mesenchymal stem cell isolation and culture methods. Cytotechnology 2013; 65:819-827

31. Tsagias N, Koliakos I, Karagiannis V, Eleftheriadou M, Koliakos GG. Isolation of mesenchymal stem cells using the total length of umbilical cord for transplantation purposes. Transfus Med 2011;21:253-261

32. Ding Y, Yang H, Feng JB, Qiu Y, Li DS, Zeng Y. Human umbilical cord-derived MSC culture: the replacement of animal sera with human cord blood plasma. In Vitro Cell Dev Biol Anim 2013;49:771-777

33. Corotchi MC, Popa MA, Remes A, Sima LE, Gussi I, Lupu Plesu M. Isolation method and xeno-free culture conditions influence multipotent differentiation capacity of human Wharton's jelly-derived mesenchymal stem cells. Stem Cell Res Ther 2013;4:81

34. Kim SM, Moon SH, Lee Y, Kim GJ, Chung HM, Choi YS. Alternative xeno-free biomaterials derived from human umbilical cord for the self-renewal ex-vivo expansion of mesenchymal stem cells. Stem Cells Dev 2013;22:3025-3038

35. Pereira T, Ivanova G, Caseiro AR, Barbosa P, Bártolo PJ, Santos JD, Luís AL, Maurício AC. MSCs conditioned me- dia and umbilical cord blood plasma metabolomics and composition. PLoS One 2014;9:e113769

36. Pham PV, Vu NB, Pham VM, Truong NH, Pham TL, Dang LT, Nguyen TT, Bui AN, Phan NK. Good manufacturing practice-compliant isolation and culture of human umbilical cord blood-derived mesenchymal stem cells. J Transl Med 2014;12:56

37. Kinzebach S, Bieback K. Expansion of Mesenchymal Stem/Stromal cells under xenogenic-free culture conditions. Adv Biochem Eng Biotechnol 2013;129:33-57

38. Swamynathan P, Venugopal P, Kannan S, Thej C, Kolkundar U, Bhagwat S, Ta M, Majumdar AS, Balasubramanian S. Are serum-free and xeno-free culture conditions ideal for large scale clinical grade expansion of Wharton's jelly derived mesenchymal stem cells? A comparative study. Stem Cell Res Ther 2014;5:88

39. Chen G, Yue A, Ruan Z, Yin Y, Wang R, Ren Y, Zhu L. Human umbilical cord-derived mesenchymal stem cells do not undergo malignant transformation during long-term culturing in serum-free medium. PLoS One 2014;9:e98565

40. Yang LM, Liu Y, Zhao J, Hao LM, Huang KX, Jiang WH. Characterization of human umbilical cord mesenchymal stem cells following tissue mass culture. Cell Mol Biol (Noisy-le-grand) 2014;60:12-18 